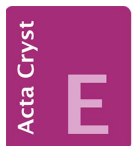

CRYSTALLOGRAPHIC COMMUNICATIONS

ISSN 2056-9890

\section{Crystal structure of pyriproxyfen}

\author{
Gihaeng Kang, Jineun Kim, ${ }^{*}$ Hyunjin Park and Tae Ho \\ Kim*
}

Department of Chemistry and Research Institute of Natural Sciences, Gyeongsang National University, Jinju 660-701, Republic of Korea. *Correspondence e-mail:

thkim@gnu.ac.kr, jekim@gnu.ac.kr

Received 7 July 2015; accepted 14 July 2015

Edited by W. T. A. Harrison, University of Aberdeen, Scotland

In the title compound \{systematic name: 4-phenoxyphenyl (RS)-2-[(pyridin-2-yl)oxy]propyl ether], $\mathrm{C}_{20} \mathrm{H}_{19} \mathrm{NO}_{3}$, which is a juvenile hormone mimic and insecticide, the dihedral angles between the plane of the central benene ring and those of the pendant pyridine ring and phenyl ring are 78.09 (6) and $82.14(8)^{\circ}$, respectively. The conformation of the $\mathrm{O}-\mathrm{C}-\mathrm{C}-$ O linkage is gauche [torsion angle $=-75.0(2)^{\circ}$ ]. In the crystal, weak aromatic $\pi-\pi$ stacking interactions [centroid-centroid separation $=3.8436(13) \AA]$ and $\mathrm{C}-\mathrm{H} \cdots \pi$ interactions link adjacent molecules, forming a three-dimensional network.

Keywords: crystal structure; pyriproxyfen; ether; juvenile hormone mimic; insecticide; $\pi-\pi$ stacking.

CCDC reference: 1412612

\section{Related literature}

For information on the insecticidal properties of the title compound, see: Shah et al. (2015). For related crystal structures, see: Ji et al. (2013); Kang et al. (2014).

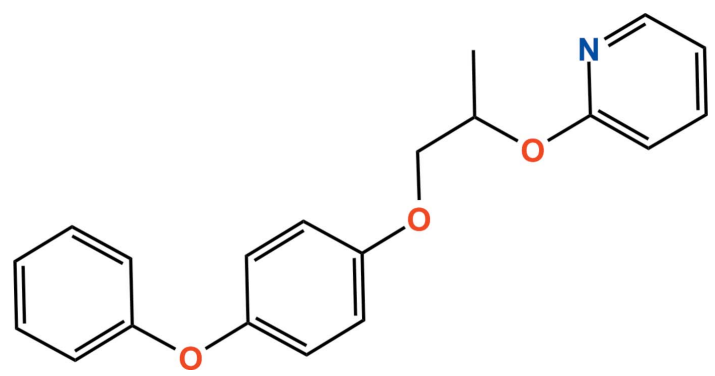

\section{Experimental}

2.1. Crystal data

$\mathrm{C}_{20} \mathrm{H}_{19} \mathrm{NO}_{3} \quad M_{r}=321.36$
Orthorhombic, Pbca

$a=10.0676(2) \AA$

$b=8.0279(1) \AA$

$c=40.9129(7) \AA$

$V=3306.65(10) \AA^{3}$

$Z=8$

Mo $K \alpha$ radiation

$\mu=0.09 \mathrm{~mm}^{-1}$

$T=173 \mathrm{~K}$

$0.25 \times 0.13 \times 0.03 \mathrm{~mm}$

\subsection{Data collection \\ Bruker APEXII CCD diffractometer \\ Absorption correction: multi-scan (SADABS; Bruker, 2009) \\ $T_{\min }=0.979, T_{\max }=0.997$}

\subsection{Refinement}

$R\left[F^{2}>2 \sigma\left(F^{2}\right)\right]=0.056$

$w R\left(F^{2}\right)=0.148$

$S=1.04$

3238 reflections

218 parameters

$\mathrm{H}$-atom parameters constrained

$\Delta \rho_{\max }=0.66 \mathrm{e} \AA^{-3}$

$\Delta \rho_{\min }=-0.25 \mathrm{e}^{-3}$

Table 1

$\mathrm{C}-\mathrm{H} \cdots \pi$ interactions $\left(\AA{ }^{\circ}\right)$.

$C g 1$ and $C g 2$ are the centroids of the $\mathrm{N} 1 / \mathrm{C} 4 / \mathrm{C} 3 / \mathrm{C} 2 / \mathrm{C} 1 / \mathrm{C} 5$ and $\mathrm{C} 15-\mathrm{C} 20$ rings, respectively.

\begin{tabular}{lllll}
\hline$D-\mathrm{H} \cdots A$ & $D-\mathrm{H}$ & $\mathrm{H} \cdots A$ & $D \cdots A$ & $D-\mathrm{H} \cdots A$ \\
\hline $\mathrm{C} 2-\mathrm{H} 2 \cdots C g 1^{\mathrm{i}}$ & 0.95 & 2.85 & $3.667(3)$ & 145 \\
$\mathrm{C} 14-\mathrm{H} 14 \cdots C g 2^{\mathrm{ii}}$ & 0.95 & 2.86 & $3.733(2)$ & 152 \\
$\mathrm{C} 19-\mathrm{H} 19 \cdots C g 2^{\text {iii }}$ & 0.95 & 2.97 & $3.857(2)$ & 156 \\
\hline
\end{tabular}

Symmetry codes: (i) $-x+\frac{3}{2}, y-\frac{1}{2}, z$; (ii) $x, y-1, z$; (iii) $-x+2, y+\frac{1}{2},-z+\frac{1}{2}$.

Data collection: APEX2 (Bruker, 2009); cell refinement: SAINT (Bruker, 2009); data reduction: $S A I N T$; $\operatorname{program}(\mathrm{s})$ used to solve structure: SHELXS97 (Sheldrick 2008); program(s) used to refine structure: SHELXL2013 (Sheldrick, 2015); molecular graphics: DIAMOND (Brandenburg, 2010); software used to prepare material for publication: SHELXTL (Sheldrick, 2008).

\section{Acknowledgements}

This research was supported by the Basic Science Research Program through the National Research Foundation of Korea (NRF) funded by the Ministry of Education, Science and Technology (No. 2015R1D1A4A01020317).

Supporting information for this paper is available from the IUCr electronic archives (Reference: HB7462).

\section{References}

Brandenburg, K. (2010). DIAMOND. Crystal Impact GbR, Bonn, Germany. Bruker (2009). APEX2, SAINT and SADABS. Bruker AXS Inc., Madison, Wisconsin, USA.

Ji, J., Zhang, X. Q., Wang, K., Ju, C. F. \& Chen, Q. (2013). Acta Cryst. E69, o547.

Kang, G., Jeon, Y., Lee, S. \& Kim, T. H. (2014). Acta Cryst. E70, o1265.

Shah, R. M., Shad, S. A. \& Abbas, N. (2015). Pestic. Biochem. Physiol. 119, $67-$ 73.

Sheldrick, G. M. (2008). Acta Cryst. A64, 112-122.

Sheldrick, G. M. (2015). Acta Cryst. C71, 3-8. 


\section{supporting information}

Acta Cryst. (2015). E71, o588 [https://doi.org/10.1107/S2056989015013481]

\section{Crystal structure of pyriproxyfen}

\section{Gihaeng Kang, Jineun Kim, Hyunjin Park and Tae Ho Kim}

\section{S1. Comment}

Pyriproxyfen, [systematic name: 4-phenoxyphenyl ( $R S$ )-2-(2-pyridyloxy)propyl ether], is the juvenile hormone mimics and it has been used for the management of many insect pests including the house fly (Shah et al., 2015). However, until now its crystal structure has not been reported. In the title compound (Fig. 1), the dihedral angles between the planes of the central benzyl ring and the terminal pyridine ring and phenyl ring system are $78.09(6)$ and $82.14(8)^{\circ}$, respectively. All bond lengths and bond angles are normal and comparable to those observed in the crystal structure of a similar compound (Ji et al., 2013; Kang et al., 2014).

In the crystal (Fig. 2), weak intermolecular $\mathrm{C}-\mathrm{H} \cdots \pi$ interactions link adjacent molecules, forming a three-dimensional network (Table. 1). In addition, weak $\pi-\pi$ interactions $\left[C g 1 \cdots C g 1^{\text {iv }}, 3.8436(13) \AA\right]$ are present $(C g 1$ is the centroid of the N1-C5 ring)[for symmetry codes: (iv), $-x+2,-y-1,-z]$.

\section{S2. Experimental}

The title compound was purchased from the Dr Ehrenstorfer GmbH Company. Slow evaporation of a solution in $\mathrm{CH}_{2} \mathrm{Cl}_{2}$ gave single crystals suitable for X-ray analysis in the form of colourless blocks.

\section{S3. Refinement}

All H-atoms were positioned geometrically and refined using a riding model with $\mathrm{d}(\mathrm{C}-\mathrm{H})=1.00 \AA, U_{\text {iso }}=1.2 U_{\text {eq }}(\mathrm{C})$ for $\mathrm{Csp}{ }^{3}-\mathrm{H}, \mathrm{d}(\mathrm{C}-\mathrm{H})=0.99 \AA, U_{\text {iso }}=1.2 U_{\text {eq }}(\mathrm{C})$ for $\mathrm{CH}_{2}$ groups, $\mathrm{d}(\mathrm{C}-\mathrm{H})=0.98 \AA, U_{\text {iso }}=1.2 U_{\text {eq }}(\mathrm{C})$ for $\mathrm{CH}_{3}$ groups, $\mathrm{d}(\mathrm{C}-$ $\mathrm{H})=0.95 \AA, U_{\text {iso }}=1.2 U_{\text {eq }}(\mathrm{C})$ for aromatic $\mathrm{C}-\mathrm{H}$.

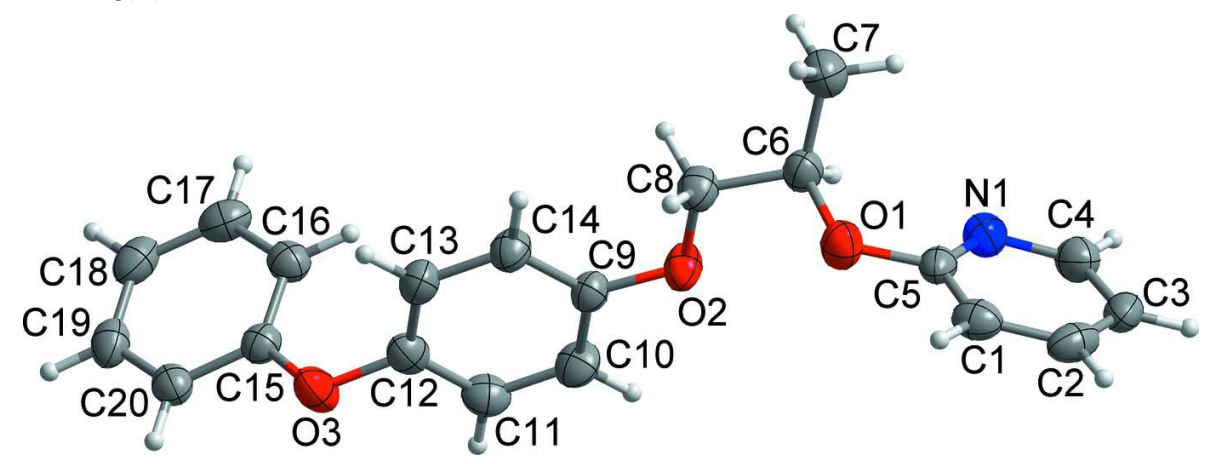

Figure 1

The asymmetric unit of the title compound with displacement ellipsoids drawn at the $50 \%$ probability level. $\mathrm{H}$ atoms are shown as small spheres of arbitrary radius. 


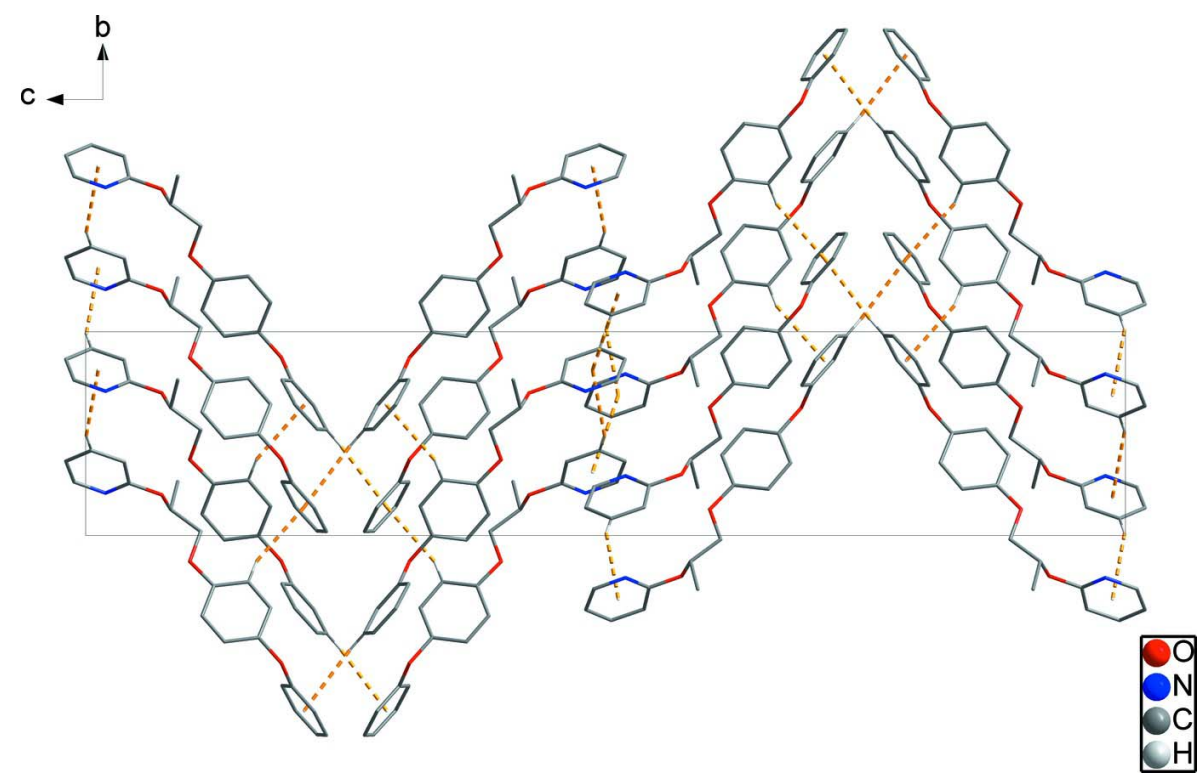

Figure 2

Crystal packing viewed along the $a$ axis. The weak $\mathrm{C}-\mathrm{H} \cdots \pi$ and $\pi-\pi$ interactions are shown as dashed lines.

4-Phenoxyphenyl (RS)-2-[(pyridin-2-yl)oxy]propyl ether

Crystal data

$\mathrm{C}_{20} \mathrm{H}_{19} \mathrm{NO}_{3}$

$M_{r}=321.36$

Orthorhombic, $\mathrm{Pbca}$

$a=10.0676(2) \AA$

$b=8.0279(1) \AA$

$c=40.9129(7) \AA$

$V=3306.65(10) \AA^{3}$

$Z=8$

$F(000)=1360$

\section{Data collection}

Bruker APEXII CCD diffractometer

$\varphi$ and $\omega$ scans

Absorption correction: multi-scan

(SADABS; Bruker, 2009)

$T_{\min }=0.979, T_{\max }=0.997$

52074 measured reflections

\section{Refinement}

Refinement on $F^{2}$

Least-squares matrix: full

$R\left[F^{2}>2 \sigma\left(F^{2}\right)\right]=0.056$

$w R\left(F^{2}\right)=0.148$

$S=1.04$

3238 reflections

218 parameters

0 restraints
$D_{\mathrm{x}}=1.291 \mathrm{Mg} \mathrm{m}^{-3}$

Mo $K \alpha$ radiation, $\lambda=0.71073 \AA$

Cell parameters from 7580 reflections

$\theta=2.3-22.0^{\circ}$

$\mu=0.09 \mathrm{~mm}^{-1}$

$T=173 \mathrm{~K}$

Block, colourless

$0.25 \times 0.13 \times 0.03 \mathrm{~mm}$

3238 independent reflections

2515 reflections with $I>2 \sigma(I)$

$R_{\text {int }}=0.065$

$\theta_{\text {max }}=26.0^{\circ}, \theta_{\min }=2.0^{\circ}$

$h=-12 \rightarrow 12$

$k=-9 \rightarrow 9$

$l=-50 \rightarrow 50$

Hydrogen site location: inferred from neighbouring sites

$\mathrm{H}$-atom parameters constrained

$w=1 /\left[\sigma^{2}\left(F_{\mathrm{o}}^{2}\right)+(0.0576 P)^{2}+2.9207 P\right]$

$$
\text { where } P=\left(F_{\mathrm{o}}^{2}+2 F_{\mathrm{c}}{ }^{2}\right) / 3
$$

$(\Delta / \sigma)_{\max }=0.001$

$\Delta \rho_{\max }=0.66 \mathrm{e} \AA^{-3}$

$\Delta \rho_{\min }=-0.25$ e $\AA^{-3}$ 


\section{Special details}

Geometry. All e.s.d.'s (except the e.s.d. in the dihedral angle between two 1.s. planes) are estimated using the full covariance matrix. The cell e.s.d.'s are taken into account individually in the estimation of e.s.d.'s in distances, angles and torsion angles; correlations between e.s.d.'s in cell parameters are only used when they are defined by crystal symmetry. An approximate (isotropic) treatment of cell e.s.d.'s is used for estimating e.s.d.'s involving l.s. planes.

Fractional atomic coordinates and isotropic or equivalent isotropic displacement parameters $\left(\AA^{2}\right)$

\begin{tabular}{|c|c|c|c|c|}
\hline & $x$ & $y$ & $z$ & $U_{\text {iso }} * / U_{\text {eq }}$ \\
\hline $\mathrm{O} 1$ & $0.93462(16)$ & $-0.1993(2)$ & $0.07340(4)$ & $0.0440(4)$ \\
\hline $\mathrm{O} 2$ & $0.97257(17)$ & $0.12811(19)$ & $0.10028(4)$ & $0.0407(4)$ \\
\hline $\mathrm{O} 3$ & $0.84983(16)$ & $0.6264(2)$ & $0.18833(4)$ & $0.0470(4)$ \\
\hline N1 & $0.9994(2)$ & $-0.2077(3)$ & $0.01922(5)$ & $0.0430(5)$ \\
\hline $\mathrm{C} 1$ & $0.8083(2)$ & $-0.3616(3)$ & $0.03714(6)$ & $0.0427(6)$ \\
\hline H1 & 0.7514 & -0.3931 & 0.0546 & $0.051^{*}$ \\
\hline $\mathrm{C} 2$ & $0.7879(2)$ & $-0.4169(3)$ & $0.00560(6)$ & $0.0454(6)$ \\
\hline $\mathrm{H} 2$ & 0.7164 & -0.4901 & 0.0008 & $0.054^{*}$ \\
\hline $\mathrm{C} 3$ & $0.8727(2)$ & $-0.3645(3)$ & $-0.01884(6)$ & $0.0446(6)$ \\
\hline $\mathrm{H} 3$ & 0.8595 & -0.3993 & -0.0408 & $0.054^{*}$ \\
\hline $\mathrm{C} 4$ & $0.9750(2)$ & $-0.2626(3)$ & $-0.01099(6)$ & $0.0443(6)$ \\
\hline $\mathrm{H} 4$ & 1.0329 & -0.2280 & -0.0280 & $0.053^{*}$ \\
\hline $\mathrm{C} 5$ & $0.9177(2)$ & $-0.2561(3)$ & $0.04197(5)$ & $0.0357(5)$ \\
\hline C6 & $1.0603(2)$ & $-0.1277(3)$ & $0.08188(5)$ & $0.0385(5)$ \\
\hline H6 & 1.0927 & -0.0579 & 0.0633 & $0.046^{*}$ \\
\hline C7 & $1.1605(3)$ & $-0.2635(3)$ & $0.08878(6)$ & $0.0516(7)$ \\
\hline H7A & 1.1273 & -0.3353 & 0.1063 & $0.077^{*}$ \\
\hline H7B & 1.2449 & -0.2131 & 0.0955 & $0.077^{*}$ \\
\hline $\mathrm{H} 7 \mathrm{C}$ & 1.1743 & -0.3300 & 0.0690 & $0.077^{*}$ \\
\hline $\mathrm{C} 8$ & $1.0369(3)$ & $-0.0192(3)$ & $0.11085(6)$ & $0.0413(6)$ \\
\hline $\mathrm{H} 8 \mathrm{~A}$ & 0.9807 & -0.0781 & 0.1270 & $0.050^{*}$ \\
\hline H8B & 1.1225 & 0.0088 & 0.1214 & $0.050^{*}$ \\
\hline C9 & $0.9480(2)$ & $0.2473(3)$ & $0.12354(5)$ & $0.0328(5)$ \\
\hline $\mathrm{C} 10$ & $0.8926(3)$ & $0.3944(3)$ & $0.11212(6)$ & $0.0418(6)$ \\
\hline H10 & 0.8755 & 0.4079 & 0.0894 & $0.050^{*}$ \\
\hline C11 & $0.8624(2)$ & $0.5218(3)$ & $0.13372(6)$ & $0.0419(6)$ \\
\hline H11 & 0.8244 & 0.6227 & 0.1260 & $0.050^{*}$ \\
\hline $\mathrm{C} 12$ & $0.8880(2)$ & $0.5010(3)$ & $0.16646(6)$ & $0.0381(5)$ \\
\hline $\mathrm{C} 13$ & $0.9423(2)$ & $0.3563(3)$ & $0.17803(6)$ & $0.0405(6)$ \\
\hline H13 & 0.9588 & 0.3437 & 0.2008 & $0.049^{*}$ \\
\hline C14 & $0.9732(2)$ & $0.2277(3)$ & $0.15657(5)$ & $0.0389(5)$ \\
\hline H14 & 1.0114 & 0.1273 & 0.1645 & $0.047^{*}$ \\
\hline $\mathrm{C} 15$ & $0.9434(2)$ & $0.7403(3)$ & $0.19842(5)$ & $0.0335(5)$ \\
\hline C16 & $1.0758(2)$ & $0.7374(3)$ & $0.18908(5)$ & $0.0376(5)$ \\
\hline H16 & 1.1078 & 0.6548 & 0.1744 & $0.045^{*}$ \\
\hline $\mathrm{C} 17$ & $1.1612(3)$ & $0.8584(3)$ & $0.20167(6)$ & $0.0441(6)$ \\
\hline H17 & 1.2521 & 0.8581 & 0.1955 & $0.053^{*}$ \\
\hline C18 & $1.1157(3)$ & $0.9779(3)$ & $0.22293(6)$ & $0.0465(6)$ \\
\hline H18 & 1.1751 & 1.0585 & 0.2316 & $0.056^{*}$ \\
\hline
\end{tabular}




\begin{tabular}{lllll}
$\mathrm{C} 19$ & $0.9830(3)$ & $0.9803(3)$ & $0.23162(5)$ & $0.0434(6)$ \\
$\mathrm{H} 19$ & 0.9510 & 1.0640 & 0.2460 & $0.052^{*}$ \\
$\mathrm{C} 20$ & $0.8970(2)$ & $0.8620(3)$ & $0.21951(5)$ & $0.0387(5)$ \\
$\mathrm{H} 20$ & 0.8060 & 0.8639 & 0.2256 & $0.046^{*}$ \\
\hline
\end{tabular}

Atomic displacement parameters $\left(\AA^{2}\right)$

\begin{tabular}{lllllll}
\hline & $U^{11}$ & $U^{22}$ & $U^{33}$ & $U^{12}$ & $U^{13}$ & $U^{23}$ \\
\hline O1 & $0.0434(10)$ & $0.0497(10)$ & $0.0389(9)$ & $-0.0070(8)$ & $0.0040(7)$ & $-0.0049(7)$ \\
O2 & $0.0592(10)$ & $0.0341(8)$ & $0.0290(8)$ & $0.0042(8)$ & $-0.0059(7)$ & $0.0004(6)$ \\
O3 & $0.0366(9)$ & $0.0428(10)$ & $0.0618(11)$ & $-0.0025(8)$ & $0.0069(8)$ & $-0.0159(8)$ \\
N1 & $0.0424(11)$ & $0.0441(12)$ & $0.0424(11)$ & $0.0004(9)$ & $0.0015(9)$ & $-0.0029(9)$ \\
C1 & $0.0396(13)$ & $0.0426(14)$ & $0.0459(14)$ & $0.0053(11)$ & $0.0021(11)$ & $0.0098(11)$ \\
C2 & $0.0387(13)$ & $0.0353(13)$ & $0.0622(16)$ & $-0.0019(11)$ & $-0.0161(12)$ & $0.0006(12)$ \\
C3 & $0.0472(14)$ & $0.0498(14)$ & $0.0368(13)$ & $0.0126(12)$ & $-0.0118(11)$ & $-0.0084(11)$ \\
C4 & $0.0430(14)$ & $0.0494(15)$ & $0.0404(13)$ & $0.0033(12)$ & $0.0011(11)$ & $0.0014(11)$ \\
C5 & $0.0401(12)$ & $0.0340(12)$ & $0.0330(11)$ & $0.0096(10)$ & $-0.0075(10)$ & $-0.0037(9)$ \\
C6 & $0.0378(13)$ & $0.0424(13)$ & $0.0353(12)$ & $-0.0025(11)$ & $-0.0014(10)$ & $-0.0053(10)$ \\
C7 & $0.0529(16)$ & $0.0536(16)$ & $0.0484(14)$ & $0.0071(13)$ & $-0.0012(12)$ & $-0.0074(12)$ \\
C8 & $0.0508(14)$ & $0.0360(13)$ & $0.0372(12)$ & $0.0029(11)$ & $-0.0045(10)$ & $-0.0006(10)$ \\
C9 & $0.0363(12)$ & $0.0306(11)$ & $0.0314(11)$ & $-0.0030(9)$ & $-0.0030(9)$ & $-0.0007(9)$ \\
C10 & $0.0506(14)$ & $0.0385(13)$ & $0.0362(12)$ & $-0.0009(11)$ & $-0.0043(11)$ & $0.0079(10)$ \\
C11 & $0.0430(14)$ & $0.0335(12)$ & $0.0493(14)$ & $0.0031(11)$ & $-0.0012(11)$ & $0.0057(11)$ \\
C12 & $0.0315(11)$ & $0.0365(12)$ & $0.0461(13)$ & $-0.0038(10)$ & $0.0016(10)$ & $-0.0065(10)$ \\
C13 & $0.0429(13)$ & $0.0452(14)$ & $0.0334(12)$ & $0.0002(11)$ & $-0.0040(10)$ & $-0.0040(10)$ \\
C14 & $0.0470(14)$ & $0.0355(12)$ & $0.0343(12)$ & $0.0029(11)$ & $-0.0064(10)$ & $0.0017(10)$ \\
C15 & $0.0376(12)$ & $0.0306(11)$ & $0.0322(11)$ & $-0.0006(10)$ & $-0.0030(9)$ & $0.0020(9)$ \\
C16 & $0.0398(13)$ & $0.0362(12)$ & $0.0368(12)$ & $0.0040(10)$ & $0.0008(10)$ & $0.0029(10)$ \\
C17 & $0.0384(13)$ & $0.0463(14)$ & $0.0477(14)$ & $-0.0033(11)$ & $-0.0069(11)$ & $0.0093(12)$ \\
C18 & $0.0561(16)$ & $0.0392(13)$ & $0.0440(13)$ & $-0.0040(12)$ & $-0.0170(12)$ & $0.0032(11)$ \\
C19 & $0.0605(16)$ & $0.0382(13)$ & $0.0314(12)$ & $0.0032(12)$ & $-0.0073(11)$ & $-0.0018(10)$ \\
C20 & $0.0436(13)$ & $0.0390(12)$ & $0.0335(12)$ & $0.0068(11)$ & $-0.0007(10)$ & $0.0010(10)$ \\
& & & & & & \\
& & & & & &
\end{tabular}

Geometric parameters $\left(\AA,{ }^{\circ}\right)$

\begin{tabular}{llll}
\hline $\mathrm{O} 1-\mathrm{C} 5$ & $1.375(3)$ & $\mathrm{C} 8-\mathrm{H} 8 \mathrm{~B}$ & 0.9900 \\
$\mathrm{O} 1-\mathrm{C} 6$ & $1.432(3)$ & $\mathrm{C} 9-\mathrm{C} 14$ & $1.384(3)$ \\
$\mathrm{O} 2-\mathrm{C} 9$ & $1.372(3)$ & $\mathrm{C} 9-\mathrm{C} 10$ & $1.387(3)$ \\
$\mathrm{O} 2-\mathrm{C} 8$ & $1.416(3)$ & $\mathrm{C} 10-\mathrm{C} 11$ & $1.386(3)$ \\
$\mathrm{O} 3-\mathrm{C} 15$ & $1.376(3)$ & $\mathrm{C} 10-\mathrm{H} 10$ & 0.9500 \\
$\mathrm{O} 3-\mathrm{C} 12$ & $1.401(3)$ & $\mathrm{C} 11-\mathrm{C} 12$ & $1.374(3)$ \\
$\mathrm{N} 1-\mathrm{C} 5$ & $1.302(3)$ & $\mathrm{C} 11-\mathrm{H} 11$ & 0.9500 \\
$\mathrm{~N} 1-\mathrm{C} 4$ & $1.335(3)$ & $\mathrm{C} 12-\mathrm{C} 13$ & $1.368(3)$ \\
$\mathrm{C} 1-\mathrm{C} 2$ & $1.380(3)$ & $\mathrm{C} 13-\mathrm{C} 14$ & $1.391(3)$ \\
$\mathrm{C} 1-\mathrm{C} 5$ & $1.404(3)$ & $\mathrm{C} 13-\mathrm{H} 13$ & 0.9500 \\
$\mathrm{C} 1-\mathrm{H} 1$ & 0.9500 & $\mathrm{C} 14-\mathrm{H} 14$ & 0.9500 \\
$\mathrm{C} 2-\mathrm{C} 3$ & $1.380(4)$ & $\mathrm{C} 15-\mathrm{C} 20$ & $1.385(3)$ \\
$\mathrm{C} 2-\mathrm{H} 2$ & 0.9500 & $\mathrm{C} 15-\mathrm{C} 16$ & $1.387(3)$
\end{tabular}




\begin{tabular}{|c|c|c|c|}
\hline $\mathrm{C} 3-\mathrm{C} 4$ & $1.354(4)$ & $\mathrm{C} 16-\mathrm{C} 17$ & $1.395(3)$ \\
\hline $\mathrm{C} 3-\mathrm{H} 3$ & 0.9500 & $\mathrm{C} 16-\mathrm{H} 16$ & 0.9500 \\
\hline $\mathrm{C} 4-\mathrm{H} 4$ & 0.9500 & $\mathrm{C} 17-\mathrm{C} 18$ & $1.373(4)$ \\
\hline $\mathrm{C} 6-\mathrm{C} 8$ & $1.490(3)$ & $\mathrm{C} 17-\mathrm{H} 17$ & 0.9500 \\
\hline $\mathrm{C} 6-\mathrm{C} 7$ & $1.511(3)$ & $\mathrm{C} 18-\mathrm{C} 19$ & $1.382(4)$ \\
\hline $\mathrm{C} 6-\mathrm{H} 6$ & 1.0000 & $\mathrm{C} 18-\mathrm{H} 18$ & 0.9500 \\
\hline $\mathrm{C} 7-\mathrm{H} 7 \mathrm{~A}$ & 0.9800 & $\mathrm{C} 19-\mathrm{C} 20$ & $1.377(3)$ \\
\hline $\mathrm{C} 7-\mathrm{H} 7 \mathrm{~B}$ & 0.9800 & C19-H19 & 0.9500 \\
\hline $\mathrm{C} 7-\mathrm{H} 7 \mathrm{C}$ & 0.9800 & $\mathrm{C} 20-\mathrm{H} 20$ & 0.9500 \\
\hline $\mathrm{C} 8-\mathrm{H} 8 \mathrm{~A}$ & 0.9900 & & \\
\hline $\mathrm{C} 5-\mathrm{O} 1-\mathrm{C} 6$ & $117.96(18)$ & $\mathrm{O} 2-\mathrm{C} 9-\mathrm{C} 14$ & $124.4(2)$ \\
\hline $\mathrm{C} 9-\mathrm{O} 2-\mathrm{C} 8$ & $116.94(16)$ & $\mathrm{O} 2-\mathrm{C} 9-\mathrm{C} 10$ & $115.65(19)$ \\
\hline $\mathrm{C} 15-\mathrm{O} 3-\mathrm{C} 12$ & $118.77(17)$ & $\mathrm{C} 14-\mathrm{C} 9-\mathrm{C} 10$ & $120.0(2)$ \\
\hline $\mathrm{C} 5-\mathrm{N} 1-\mathrm{C} 4$ & $116.5(2)$ & $\mathrm{C} 11-\mathrm{C} 10-\mathrm{C} 9$ & $120.1(2)$ \\
\hline $\mathrm{C} 2-\mathrm{C} 1-\mathrm{C} 5$ & $116.3(2)$ & $\mathrm{C} 11-\mathrm{C} 10-\mathrm{H} 10$ & 119.9 \\
\hline $\mathrm{C} 2-\mathrm{C} 1-\mathrm{H} 1$ & 121.9 & $\mathrm{C} 9-\mathrm{C} 10-\mathrm{H} 10$ & 119.9 \\
\hline $\mathrm{C} 5-\mathrm{C} 1-\mathrm{H} 1$ & 121.9 & $\mathrm{C} 12-\mathrm{C} 11-\mathrm{C} 10$ & $119.4(2)$ \\
\hline $\mathrm{C} 1-\mathrm{C} 2-\mathrm{C} 3$ & $119.1(2)$ & $\mathrm{C} 12-\mathrm{C} 11-\mathrm{H} 11$ & 120.3 \\
\hline $\mathrm{C} 1-\mathrm{C} 2-\mathrm{H} 2$ & 120.4 & $\mathrm{C} 10-\mathrm{C} 11-\mathrm{H} 11$ & 120.3 \\
\hline $\mathrm{C} 3-\mathrm{C} 2-\mathrm{H} 2$ & 120.4 & $\mathrm{C} 13-\mathrm{C} 12-\mathrm{C} 11$ & $121.0(2)$ \\
\hline $\mathrm{C} 4-\mathrm{C} 3-\mathrm{C} 2$ & $118.9(2)$ & $\mathrm{C} 13-\mathrm{C} 12-\mathrm{O} 3$ & $119.9(2)$ \\
\hline $\mathrm{C} 4-\mathrm{C} 3-\mathrm{H} 3$ & 120.6 & $\mathrm{C} 11-\mathrm{C} 12-\mathrm{O} 3$ & $118.9(2)$ \\
\hline $\mathrm{C} 2-\mathrm{C} 3-\mathrm{H} 3$ & 120.6 & $\mathrm{C} 12-\mathrm{C} 13-\mathrm{C} 14$ & $120.1(2)$ \\
\hline $\mathrm{N} 1-\mathrm{C} 4-\mathrm{C} 3$ & $124.0(2)$ & $\mathrm{C} 12-\mathrm{C} 13-\mathrm{H} 13$ & 119.9 \\
\hline $\mathrm{N} 1-\mathrm{C} 4-\mathrm{H} 4$ & 118.0 & $\mathrm{C} 14-\mathrm{C} 13-\mathrm{H} 13$ & 119.9 \\
\hline $\mathrm{C} 3-\mathrm{C} 4-\mathrm{H} 4$ & 118.0 & $\mathrm{C} 9-\mathrm{C} 14-\mathrm{C} 13$ & $119.4(2)$ \\
\hline $\mathrm{N} 1-\mathrm{C} 5-\mathrm{O} 1$ & $119.5(2)$ & $\mathrm{C} 9-\mathrm{C} 14-\mathrm{H} 14$ & 120.3 \\
\hline $\mathrm{N} 1-\mathrm{C} 5-\mathrm{C} 1$ & $125.1(2)$ & $\mathrm{C} 13-\mathrm{C} 14-\mathrm{H} 14$ & 120.3 \\
\hline $\mathrm{O} 1-\mathrm{C} 5-\mathrm{C} 1$ & $115.4(2)$ & $\mathrm{O} 3-\mathrm{C} 15-\mathrm{C} 20$ & $115.1(2)$ \\
\hline $\mathrm{O} 1-\mathrm{C} 6-\mathrm{C} 8$ & $106.72(19)$ & $\mathrm{O} 3-\mathrm{C} 15-\mathrm{C} 16$ & $124.4(2)$ \\
\hline $\mathrm{O} 1-\mathrm{C} 6-\mathrm{C} 7$ & $110.2(2)$ & $\mathrm{C} 20-\mathrm{C} 15-\mathrm{C} 16$ & $120.5(2)$ \\
\hline $\mathrm{C} 8-\mathrm{C} 6-\mathrm{C} 7$ & $112.2(2)$ & $\mathrm{C} 15-\mathrm{C} 16-\mathrm{C} 17$ & $118.6(2)$ \\
\hline $\mathrm{O} 1-\mathrm{C} 6-\mathrm{H} 6$ & 109.2 & $\mathrm{C} 15-\mathrm{C} 16-\mathrm{H} 16$ & 120.7 \\
\hline $\mathrm{C} 8-\mathrm{C} 6-\mathrm{H} 6$ & 109.2 & $\mathrm{C} 17-\mathrm{C} 16-\mathrm{H} 16$ & 120.7 \\
\hline $\mathrm{C} 7-\mathrm{C} 6-\mathrm{H} 6$ & 109.2 & $\mathrm{C} 18-\mathrm{C} 17-\mathrm{C} 16$ & $121.0(2)$ \\
\hline $\mathrm{C} 6-\mathrm{C} 7-\mathrm{H} 7 \mathrm{~A}$ & 109.5 & $\mathrm{C} 18-\mathrm{C} 17-\mathrm{H} 17$ & 119.5 \\
\hline $\mathrm{C} 6-\mathrm{C} 7-\mathrm{H} 7 \mathrm{~B}$ & 109.5 & $\mathrm{C} 16-\mathrm{C} 17-\mathrm{H} 17$ & 119.5 \\
\hline $\mathrm{H} 7 \mathrm{~A}-\mathrm{C} 7-\mathrm{H} 7 \mathrm{~B}$ & 109.5 & $\mathrm{C} 17-\mathrm{C} 18-\mathrm{C} 19$ & $119.6(2)$ \\
\hline $\mathrm{C} 6-\mathrm{C} 7-\mathrm{H} 7 \mathrm{C}$ & 109.5 & $\mathrm{C} 17-\mathrm{C} 18-\mathrm{H} 18$ & 120.2 \\
\hline $\mathrm{H} 7 \mathrm{~A}-\mathrm{C} 7-\mathrm{H} 7 \mathrm{C}$ & 109.5 & $\mathrm{C} 19-\mathrm{C} 18-\mathrm{H} 18$ & 120.2 \\
\hline $\mathrm{H} 7 \mathrm{~B}-\mathrm{C} 7-\mathrm{H} 7 \mathrm{C}$ & 109.5 & $\mathrm{C} 20-\mathrm{C} 19-\mathrm{C} 18$ & $120.4(2)$ \\
\hline $\mathrm{O} 2-\mathrm{C} 8-\mathrm{C} 6$ & $108.52(18)$ & $\mathrm{C} 20-\mathrm{C} 19-\mathrm{H} 19$ & 119.8 \\
\hline $\mathrm{O} 2-\mathrm{C} 8-\mathrm{H} 8 \mathrm{~A}$ & 110.0 & $\mathrm{C} 18-\mathrm{C} 19-\mathrm{H} 19$ & 119.8 \\
\hline $\mathrm{C} 6-\mathrm{C} 8-\mathrm{H} 8 \mathrm{~A}$ & 110.0 & $\mathrm{C} 19-\mathrm{C} 20-\mathrm{C} 15$ & $119.9(2)$ \\
\hline $\mathrm{O} 2-\mathrm{C} 8-\mathrm{H} 8 \mathrm{~B}$ & 110.0 & $\mathrm{C} 19-\mathrm{C} 20-\mathrm{H} 20$ & 120.1 \\
\hline $\mathrm{C} 6-\mathrm{C} 8-\mathrm{H} 8 \mathrm{~B}$ & 110.0 & $\mathrm{C} 15-\mathrm{C} 20-\mathrm{H} 20$ & 120.1 \\
\hline $\mathrm{H} 8 \mathrm{~A}-\mathrm{C} 8-\mathrm{H} 8 \mathrm{~B}$ & 108.4 & & \\
\hline
\end{tabular}




$\begin{array}{llll}\mathrm{C} 5-\mathrm{C} 1-\mathrm{C} 2-\mathrm{C} 3 & -1.1(3) & \mathrm{C} 10-\mathrm{C} 11-\mathrm{C} 12-\mathrm{C} 13 & -0.3(4) \\ \mathrm{C} 1-\mathrm{C} 2-\mathrm{C} 3-\mathrm{C} 4 & 1.2(4) & \mathrm{C} 10-\mathrm{C} 11-\mathrm{C} 12-\mathrm{O} 3 & -176.2(2) \\ \mathrm{C} 5-\mathrm{N} 1-\mathrm{C} 4-\mathrm{C} 3 & -0.5(4) & \mathrm{C} 15-\mathrm{O} 3-\mathrm{C} 12-\mathrm{C} 13 & 86.0(3) \\ \mathrm{C} 2-\mathrm{C} 3-\mathrm{C} 4-\mathrm{N} 1 & -0.4(4) & \mathrm{C} 15-\mathrm{O} 3-\mathrm{C} 12-\mathrm{C} 11 & -98.0(3) \\ \mathrm{C} 4-\mathrm{N} 1-\mathrm{C} 5-\mathrm{O} 1 & -177.7(2) & \mathrm{C} 11-\mathrm{C} 12-\mathrm{C} 13-\mathrm{C} 14 & 0.4(4) \\ \mathrm{C} 4-\mathrm{N} 1-\mathrm{C} 5-\mathrm{C} 1 & 0.7(3) & \mathrm{O} 3-\mathrm{C} 12-\mathrm{C} 13-\mathrm{C} 14 & 176.3(2) \\ \mathrm{C} 6-\mathrm{O} 1-\mathrm{C} 5-\mathrm{N} 1 & -17.0(3) & \mathrm{O} 2-\mathrm{C} 9-\mathrm{C} 14-\mathrm{C} 13 & -179.0(2) \\ \mathrm{C} 6-\mathrm{O} 1-\mathrm{C} 5-\mathrm{C} 1 & 164.4(2) & \mathrm{C} 10-\mathrm{C} 9-\mathrm{C} 14-\mathrm{C} 13 & 0.2(3) \\ \mathrm{C} 2-\mathrm{C} 1-\mathrm{C} 5-\mathrm{N} 1 & 0.1(3) & \mathrm{C} 12-\mathrm{C} 13-\mathrm{C} 14-\mathrm{C} 9 & -0.4(4) \\ \mathrm{C} 2-\mathrm{C} 1-\mathrm{C} 5-\mathrm{O} 1 & 178.6(2) & \mathrm{C} 12-\mathrm{O} 3-\mathrm{C} 15-\mathrm{C} 20 & 178.8(2) \\ \mathrm{C} 5-\mathrm{O} 1-\mathrm{C} 6-\mathrm{C} 8 & 157.85(19) & \mathrm{C} 12-\mathrm{O}-\mathrm{C} 15-\mathrm{C} 16 & -1.8(3) \\ \mathrm{C} 5-\mathrm{O} 1-\mathrm{C} 6-\mathrm{C} 7 & -80.0(2) & \mathrm{O} 3-\mathrm{C} 15-\mathrm{C} 16-\mathrm{C} 17 & -178.6(2) \\ \mathrm{C} 9-\mathrm{O} 2-\mathrm{C} 8-\mathrm{C} 6 & -177.21(19) & \mathrm{C} 20-\mathrm{C} 15-\mathrm{C} 16-\mathrm{C} 17 & 0.7(3) \\ \mathrm{O} 1-\mathrm{C} 6-\mathrm{C} 8-\mathrm{O} 2 & -75.0(2) & \mathrm{C} 15-\mathrm{C} 16-\mathrm{C} 17-\mathrm{C} 18 & 0.1(3) \\ \mathrm{C} 7-\mathrm{C} 6-\mathrm{C} 8-\mathrm{O} 2 & 164.1(2) & \mathrm{C} 16-\mathrm{C} 17-\mathrm{C} 18-\mathrm{C} 19 & -1.0(4) \\ \mathrm{C} 8-\mathrm{O} 2-\mathrm{C} 9-\mathrm{C} 14 & -4.7(3) & \mathrm{C} 17-\mathrm{C} 18-\mathrm{C} 19-\mathrm{C} 20 & 1.1(3) \\ \mathrm{C} 8-\mathrm{O} 2-\mathrm{C} 9-\mathrm{C} 10 & 176.0(2) & \mathrm{C} 18-\mathrm{C} 19-\mathrm{C} 20-\mathrm{C} 15 & -0.2(3) \\ \mathrm{O} 2-\mathrm{C} 9-\mathrm{C} 10-\mathrm{C} 11 & 179.2(2) & \mathrm{O} 3-\mathrm{C} 15-\mathrm{C} 20-\mathrm{C} 19 & 178.7(2) \\ \mathrm{C} 14-\mathrm{C} 9-\mathrm{C} 10-\mathrm{C} 11 & -0.1(4) & \mathrm{C} 16-\mathrm{C} 15-\mathrm{C} 20-\mathrm{C} 19 & -0.7(3) \\ \mathrm{C} 9-\mathrm{C} 10-\mathrm{C} 11-\mathrm{C} 12 & 0.1(4) & & \end{array}$

Hydrogen-bond geometry $\left(\AA,{ }^{\circ}\right)$

$\mathrm{Cg} 1$ and $\mathrm{Cg} 2$ are the centroids of the $\mathrm{N} 1 / \mathrm{C} 4 / \mathrm{C} 3 / \mathrm{C} 2 / \mathrm{C} 1 / \mathrm{C} 5$ and $\mathrm{C} 15-\mathrm{C} 20$ rings, respectively.

\begin{tabular}{lllll}
\hline$D-\mathrm{H} \cdots A$ & $D-\mathrm{H}$ & $\mathrm{H} \cdots A$ & $D \cdots A$ & $D-\mathrm{H} \cdots A$ \\
\hline $\mathrm{C} 2-\mathrm{H} 2 \cdots C g 1^{\mathrm{i}}$ & 0.95 & 2.85 & $3.667(3)$ & 145 \\
$\mathrm{C} 14-\mathrm{H} 14 \cdots C g 2^{\mathrm{ii}}$ & 0.95 & 2.86 & $3.733(2)$ & 152 \\
$\mathrm{C} 19-\mathrm{H} 19 \cdots C g 2^{\mathrm{iii}}$ & 0.95 & 2.97 & $3.857(2)$ & 156 \\
\hline
\end{tabular}

Symmetry codes: (i) $-x+3 / 2, y-1 / 2, z$; (ii) $x, y-1, z$; (iii) $-x+2, y+1 / 2,-z+1 / 2$. 